\title{
Dielectric Relaxation Spectroscopy of Aqueous Micellar Electrolyte Solutions: A Novel Application to Infer Dukhin Number and Zeta Potential of a Micelle
}

\author{
Mahmoud Khademi and Dominik P. J. Barz* \\ Department of Chemical Engineering, Queen's University, Kingston, Ontario, Canada
}

\begin{abstract}
The complex permittivities of aqueous sodium dodecyl sulfate (SDS) solutions, with and without the addition of sodium chloride $(\mathrm{NaCl})$, are measured in the frequency range from $200 \mathrm{MHz}$ to $14 \mathrm{GHz}$. The SDS concentrations are chosen such that the SDS molecules aggregate to micelles. In this frequency range, the measured spectra allow for the identification of two different relaxation processes. That is, the relaxation of the water molecules at frequencies above $1 \mathrm{GHz}$ and the micellar relaxation at frequencies lower than $1 \mathrm{GHz}$. It is found that the addition of $\mathrm{NaCl}$ to the system mostly affects the micellar relaxation process. In detail, the time constant as well as the amplitude of the relaxation decrease by adding $\mathrm{NaCl}$. These effects are attributed to the change in the solution conductivity which changes the properties of the micelle's electrical double layer. We also extract the Dukhin number of the micelles as a function of surfactant and electrolyte content from the measurements. The Dukhin number is a dimensionless group that describes the influence of the surface conductivity on a phenomena. A regression between Dukhin numbers and free sodium ions is found so that all data collapses on a single curve independent of the surfactant concentration. The surface conductivity is a manifestation of the electrical double layer and we use the Bikerman equation to infer the zeta potential of the micelles. Comparison to literature data shows very good agreement and proves that dielectric relaxation spectroscopy can be engaged to infer the zeta potential of micelles.
\end{abstract}

Keywords: Dielectric Relaxation Spectroscopy, Surfactant, Micelle, Dukhin Number, Zeta Potential

\section{Introduction}

Dielectric relaxation spectroscopy (DRS) measures the polarization of a sample in an AC electric field over a range of frequencies. It is a powerful and noninvasive tool to explore the relaxation behavior of systems having dipole moments, as well as, to study the dielectric behavior of colloidal and biological systems [1-6]. Among these systems, aqueous micellar solutions, made by the addition of surfactant molecules, are of particular interest. This is due to their significance in numerous areas such as stabilization of colloidal systems [7-9] and emulsions [10,11], coatings [12], detergents [7], to name a few. The equilibrium and dynamics of such

\footnotetext{
*E-mail: dominik.barz@queensu.ca
} 
systems are determined by the balance of several mutually-influencing phenomena. In an aqueous surfactant system with no electrolyte added, the electrostatic interactions of the (charged) surfactant heads, the dipole moment of the water molecules, the dissociated counter-ions, along with the hydrophobic interactions of the surfactant hydrocarbon tails, are the most important interactions involved in the system [13, 14].

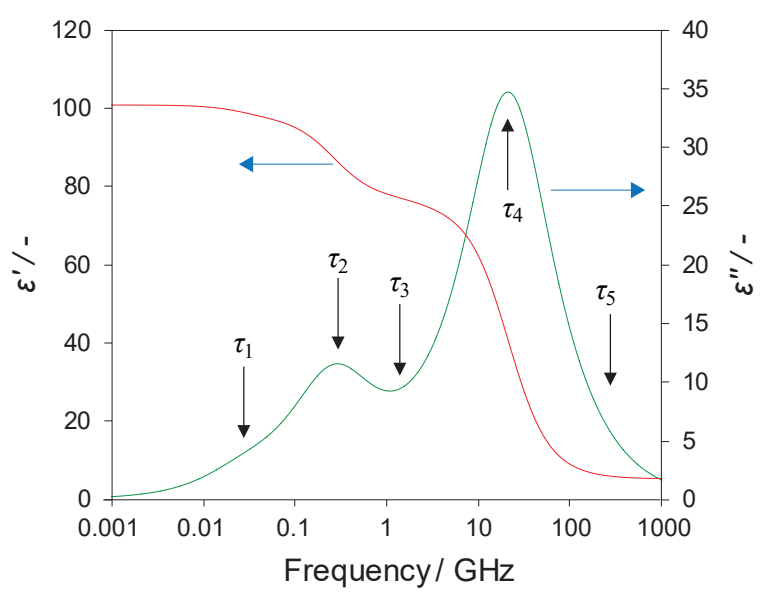

FIGURE 1: A typical dielectric relaxation spectrum of a micellar aqueous SDS solution.

Figure 1 shows a typical DRS spectrum of an aqueous micellar sodium dodecyl sulfate (SDS) solution, computed using the model provided in the Theory section, based on data of different relaxation processes described in the literature [14-16]. This spectrum features five different frequency processes $(i=1-5)$. These frequency modes are distinguished by their time scales $\tau_{i}$, or the corresponding angular frequencies, and the amplitude of the relaxation process $\Delta \varepsilon_{i}$. A review of DRS studies on micellar systems clarifies that two of these modes are associated with different micellar relaxation phenomena while the other three modes are related to the water molecules, cf. Refs $[4,14,15,17-19]$. In detail, two micellar relaxation processes $(i=$ 1,2) can be properly explained by applying the Grosse and Foster theory for colloidal particles to micelles $[20,21]$. The slowest relaxation process, having a typical relaxation time $\tau_{1}$ of several nanoseconds, is related to the fluctuation of the ion cloud around the micelle. The second relaxation process has a typical time scale of $\tau_{2} \simeq 500 \mathrm{ps}$ and can be attributed to the tangential counter-ion motion around the micelles which causes interfacial polarization $[14,16]$. At higher frequencies, the relaxation of the water molecules determines the spectrum and there are three different relaxation modes reported in the literature $(i=3-5)$. First, a dispersion step with a relaxation time of $\tau_{3} \simeq 100$ ps takes places which can be attributed to slow water molecules surrounding the hydrophobic groups of the surfactant [17, 22]. The second and most dominant dispersion step of the water molecules is related to the hydrogen bond relaxation which has a relaxation time $\tau_{4} \approx 8$ ps at room temperature. Finally, the third and fastest relaxation mode of the water molecules occurs at very high frequencies above $100 \mathrm{GHz}$. It has a typical time scale of $\tau_{5} \simeq 1 \mathrm{ps}$ and is related to the rotational relaxation 
of water molecules with one hydrogen bond or less $[17,23,24]$. It is hypothesized that water molecules are in constant change of the hydrogen bond network. For a water molecule to change its hydrogen bond network, it needs to be released from the existent network and joins and reorients itself in a new network. The release process is affiliated with $\tau_{4}$ and $\Delta \varepsilon_{4}$ while the reorientation process results in $\tau_{5}$ and $\Delta \varepsilon_{5}$ in the DRS spectrum. Consequently, the latter two relaxation processes are considered as bulk water relaxation [17, 25].

Micellar relaxation processes, i.e., $i=1$ and 2, are mainely based on the difference in the permittivity of the micelles and the surrounding medium; i.e., on an interface with a permittivity jump. The characteristics of this interfacial region determine the dispersion step. In other word, the permittivity, size and the surface conductivity of the micelles as well as the permittivity and ion content of the medium, are the determining parameters. The radial ion motion around the micelles $(i=1)$, which takes place within the diffuse part of the micelle's electrical double layer (EDL), is very sensitive to the conductivity of the medium. The second relaxation process $(i=2)$, arising from the tangential motion of the ions around the micelle, is related to the stagnant layer of the EDL. Within this region, ion content is mainly based on bound counter-ions as well as specifically adsorbed ions. Both diffuse and stagnant parts of the EDL constitute the surface conductivity [26].

DRS is a versatile method to study the ionic and dipole interactions and dynamics and it is applicable to any electrolyte in any solvent [3]. There is a lot of information that can be inferred from DRS measurements, including the permittivity of the liquid [27], ion pairs detection [2], ion solvation [2], or thermodynamics of the solvents and ions [3], just to name a few. Although there is some literature concerned with the application of DRS to micellar systems, such as Ref. [4, 14-19, 22, 28, 29], we are not aware of any attempt to use DRS to study these micellar systems in the presence of an additional electrolyte. However, an additional electrolyte is usually the case for the majority of surfactant solutions. Furthermore, we are not aware of any work that employs DRS to measure the main parameter of all electrokinetic phenomena; that is, the zeta potential. Hence, the aim of this research is first and foremost to study the relaxation processes of the micellar system in the microwave range of frequency with and without the presence of an additional electrolyte. From this knowledge, we develop a procedure to infer the zeta potential of the micelles. We choose aqueous solutions with the anionic surfactant SDS along with sodium chloride $(\mathrm{NaCl})$ as an exemplary micellar system. This article continues with a brief section on theory of the complex permittivity that is followed by the description of materials and experimental methodology. Afterwards, we report and discuss our findings based on the regression of the measured spectra to a complex permittivity model. The knowledge from our experiments is utilized to find a correlation for the Dukhin Number of a micelle as a function of the ion content. Subsequently, we propose a method to extract the zeta potentials of the micelles using the values of surface conductivities based on the Bikerman equation. Finally, the article is concluded with a summary. 


\section{Theory}

The complex (relative) permittivity $\varepsilon^{*}(\omega)$ at an angular frequency $\omega$ is defined as the ratio of the electric displacement $\mathbf{D}$ and the electric field $\mathbf{E}$, according to $\mathbf{D}=\varepsilon_{0} \varepsilon^{*}(\omega) \mathbf{E}$, where $\varepsilon_{0}$ is the permittivity of vacuum. Here, the complex permittivity consists of a real $\varepsilon^{\prime}(\omega)$ and an imaginary part $\varepsilon^{\prime \prime}(\omega)$ as indicated by the imaginary unit $j$; i.e., $\varepsilon^{*}(\omega)=\varepsilon^{\prime}(\omega)-j \varepsilon^{\prime \prime}(\omega)$. Dipole fluctuations and intramolecular polarizability are the determining parameters of the complex permittivity. Accordingly, $\varepsilon^{\prime}(\omega)$ is a measure of how molecular dipoles keep pace with an applied electric field, while $\varepsilon^{\prime \prime}(\omega)$ represents the energy dissipation in the system [2]. Dielectric relaxation spectroscopy does not directly measure the complex permittivity of a system. The measured quantity is the total loss $\eta$ which is the sum of the complex permittivity and the loss associated with the ionic DC conductivity $\sigma_{D C}$ at lower frequencies, that is

$$
\eta(\omega)=\varepsilon^{*}(\omega)+\frac{\sigma_{D C}}{j \omega \varepsilon_{0}} .
$$

There are various models to obtain the complex permittivity from a regression of the total loss data such as the Debye, Cole-Cole, Cole-Davidson, and Havriliak-Negami models. The latter one can be used for multiple relaxation processes and can be written as

$$
\varepsilon^{*}(\omega)=\varepsilon_{\infty}+\sum_{i}^{n} \frac{\Delta \varepsilon_{i}}{\left(1+\left(j \omega \tau_{i}\right)^{1-\alpha_{i}}\right)^{\beta_{i}}} .
$$

Here, $\varepsilon_{\infty}$ is the permittivity at high frequencies which is based only on the intramolecular polarizability [17]; $\Delta \varepsilon_{i}$ and $\tau_{i}$ are the dispersion amplitude and relaxation time of the $i^{\text {th }}$ relaxation process; and $\alpha_{i}$ and $\beta_{i}$ are the respective shape factors of the relaxation distribution and can have values between 0 and 1 . The HavriliakNegami model is reduced to the Debye model for $\alpha_{i}=0$ and $\beta_{i}=1$ while the Cole-Cole model is obtained in the case of $0 \leq \alpha_{i}<1$ and $\beta_{i}=1$. For $\alpha_{i}=0$ and $0<\beta_{i} \leq 1$, the Cole-Davidson model is provided $[30,31]$.

In the frequency range studied in this work, we find that the best regression quality is generally achieved with a Cole-Cole model incorporating two relaxations. That is, the tangential micellar and the water molecules hydrogen bond relaxations. As mentioned in the Section Introduction, there is another relaxation process for aqueous surfactant solutions with a relaxation time of about $100 \mathrm{ps}(i=3)$ reported in the literature $[17,28]$. However, all of our attempts to incorporate this relaxation process in our regression analysis result in a diminished degree of the coefficient of determination of the fitted parameters. Therefore, we consider only the two relaxation processes, which can be clearly identified within the frequency range, in the model for the 
benefit of a higher accuracy. Hence, the final equation that we use for the interpretation of the spectra is

$$
\eta(\omega)=\varepsilon_{\infty}+\frac{\Delta \varepsilon_{2}}{1+\left(j \omega \tau_{2}\right)^{1-\alpha_{2}}}+\frac{\Delta \varepsilon_{4}}{1+\left(j \omega \tau_{4}\right)^{1-\alpha_{4}}}+\frac{\sigma_{D C}}{j \omega \varepsilon_{0}}
$$

where the tangential micellar and the water hydrogen bond relaxation processes are denoted by subscripts 2 and 4 , respectively; cf. Figure 1.

\section{Materials and Experimental Methodology}

In this section, details of the chemicals, the experimental procedure as well as of the data analysis are presented.

\subsection{Materials}

The anionic surfactant sodium dodecyl sulfate $\left(\mathrm{NaC}_{12} \mathrm{H}_{25} \mathrm{SO}_{4}, \geq 95.0 \%\right.$, MP Biomedicals, France), sodium chloride ( $\mathrm{NaCl}, \geq 99.5 \%$, Sigma-Aldrich, Canada) along with deionized (DI) water (RiOs-DI3 water purification system, EMD Milipore Corporation, MA, USA) with a conductivity $<1 \mu \mathrm{S} / \mathrm{cm}$ are used to prepare the aqueous surfactant-electrolyte solutions.

\subsection{Experimental Methodology}

The dielectric relaxation measurements of the surfactant-electrolyte solutions are performed using a setup consisting of a dielectric probe kit (85070E, Agilent Technologies, CA, USA) and a vector network analyzer (N5247A, Agilent Technologies, CA, USA). This coaxial high temperature probe is applicable for a frequency range of $200 \mathrm{MHz}$ to $20 \mathrm{GHz}$. The vector network analyzer records the polarization of the system and reports it as the real and imaginary parts of the total loss. The measurements are performed at $22{ }^{\circ} \mathrm{C}$ and in the frequency range of $200 \mathrm{MHz}$ up to $14 \mathrm{GHz}$. Prior to conduct the DRS measurements, the experimental setup is calibrated using a DI water sample, air and short circuiting. The surfactant concentrations are chosen to always be above the critical micelle concentration (CMC), which is around $8 \mathrm{mM}$ for our experimental conditions. Therefore, the respective relaxation processes can be attributed to the micelles rather than to the free surfactant molecules. With respect to the analysis of the dielectric relaxation spectra data, we take advantage of the mathematical analogy of Equation (3) and the impedance of an equivalent electrical circuit for which a variety of powerful complex, multivariable and nonlinear regression software is available. Details of the data analysis can be found in the Supporting Information to this article. 
For verification of the conductivity values obtained from the regression procedure, we conduct independent conductivity measurements of the samples using a $\mathrm{pH}$ and conductivity meter (SevenExcellence $\mathrm{pH}$ meter system, Mettler-Toledo, Switzerland). The obtained results can be found in the Supporting Information. We find a very good agreement between the measured and the modeled conductivity values. It should be noted that we use the values from the regression throughout the article.

\section{Results}

In this section, the results of the complex permittivity measurements for aqueous solutions of SDS, as well as SDS and $\mathrm{NaCl}$ are discussed. To verify our experimental and theoretical methodology, we first measure the dielectric relaxation of DI water and compare it to literature values, cf. supporting information. We conclude that the regression of the Debye model gives very accurate results for the dielectric relaxation of pure water in the current frequency range. Hence, we can use the inferred value $\varepsilon_{\infty}=5.25$ as a constant for the samples that contain surfactants.

\subsection{Aqueous Micellar Suspensions of SDS and NaCl}

Further dielectric relaxations measurements are performed with samples containing varying amounts of SDS with and without $\mathrm{NaCl}$. First, we measure SDS samples with concentrations of 20, 35, 70, 105, 140 and 350 $\mathrm{mM}$ without addition of $\mathrm{NaCl}$. The real and imaginary parts of the total dielectric loss for three exemplary samples, along with the result of the regression to Equation (3), are shown in Figure S2 of the Supporting Information. We find that the DC conductivity loss has a considerable influence on the spectra especially at frequencies lower than $1 \mathrm{GHz}$. The respective complex permittivity spectra are obtained by subtraction of the DC conductivity loss term from the total loss, cf. Equation (1), and shown in Figure 2. Compared to the spectra of pure water, another relaxation process can be recognized, especially for the highest surfactant concentration. Here again, the hydrogen bond relaxation mode $(i=4)$ is the mode that dominates the spectra at frequencies higher than $1 \mathrm{GHz}$. In addition, another relaxation mode is visible below $1 \mathrm{GHz}$. This mode is not observed for pure water and related to the tangential micellar relaxation $(i=2)[14,15,18]$. We give all parameters of the data regression to Equation (3) for SDS samples with concentrations of 20, 35, 70, 105, 140 and $350 \mathrm{mM}$ in the Supporting Information of this article. The fitted data shows that the water molecules hydrogen bond relaxation process $(i=4)$ is hardly influenced by the variation of the surfactant concentration. The other parameters are discussed in following sections.

In addition, the effect of $\mathrm{NaCl}$ addition on the DRS spectra of aqueous SDS solutions is investigated. 


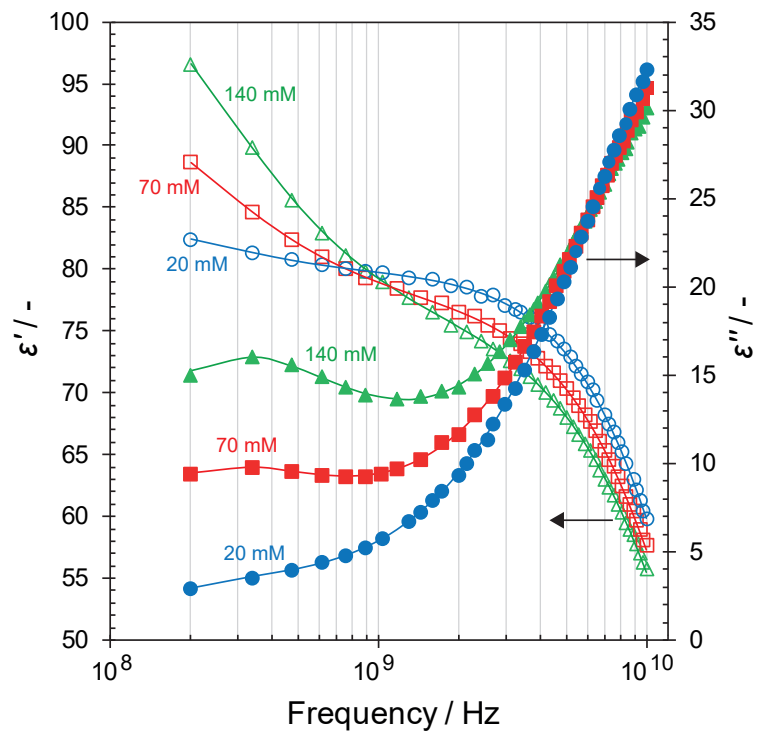

FIGURE 2: Real (open symbols) and imaginary (solid symbols) parts of the complex permittivity of three SDS solutions. Solid line are results from the data regression.

Figure 3 shows the real and imaginary parts of the measured complex permittivity (open and solid symbols, respectively) for samples with $140 \mathrm{mM}$ SDS and different $\mathrm{NaCl}$ concentrations ranging from 0 to $100 \mathrm{mM}$. The results based on regression of Equation (3) are provided as solid lines. The $\mathrm{NaCl}$ addition has mainly an influence on the tangential micellar relaxation which leads to lower real and imaginary permittivity values. The higher the $\mathrm{NaCl}$ concentration, the lower are the magnitudes at a given frequency. Additionally, we find that the $\mathrm{NaCl}$ somewhat shifts the tangential micellar relaxation peak $(i=2)$ toward higher frequencies. We give the regression parameters for different surfactant concentrations in the presence of different $\mathrm{NaCl}$ concentrations in Supporting Information. Again, we do not see a major influence of the $\mathrm{NaCl}$ addition on the relaxation process $i=4$.

The dielectric relaxation of aqueous $\mathrm{NaCl}$ solutions without surfactant in a frequency range from $20 \mathrm{MHz}$ to $40 \mathrm{GHz}$ features only one relaxation peak [31-34]. This is different for the samples containing surfactants but no $\mathrm{NaCl}$ where up to 4 different relaxation processes are reported in this frequency range [4, 14-18, 28, 35].

As presented in the permittivity spectra in Figures 2 and 3, the tangential micellar relaxation mode $(i=$ 2 ) is mainly affected by the $\mathrm{SDS}$ and $\mathrm{NaCl}$ concentration. Hence, the values of $\tau_{2}$ and $\Delta \varepsilon_{2}$ versus these concentrations are plotted in Figures 4(a)-(d) to clarify the explicit influence. Figure 4(a) shows the effect of the surfactant concentration on the relaxation time $\tau_{2}$ without and with addition $\mathrm{NaCl}$. The values of $\tau_{2}$ are observed to be relatively similar for a given $\mathrm{NaCl}$ concentration, independent of the surfactant concentration. The small deviations are mainly within the standard deviation of the measurements. Figure 4(b) shows the influence of the surfactant concentration on the relaxation amplitude $\Delta \varepsilon_{2}$. For all $\mathrm{NaCl}$ concentrations, there 


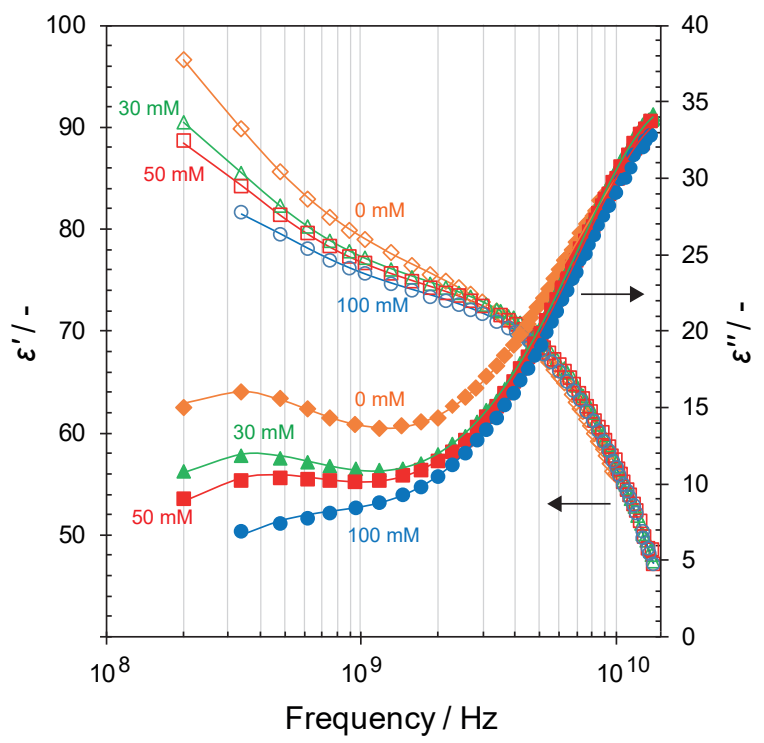

FIGURE 3: Real (open symbols) and imaginary (solid symbols) parts of the complex permittivity of $140 \mathrm{mM}$ SDS solutions with varying $\mathrm{NaCl}$ concentration. Solid line are results from the data regression.

(a)

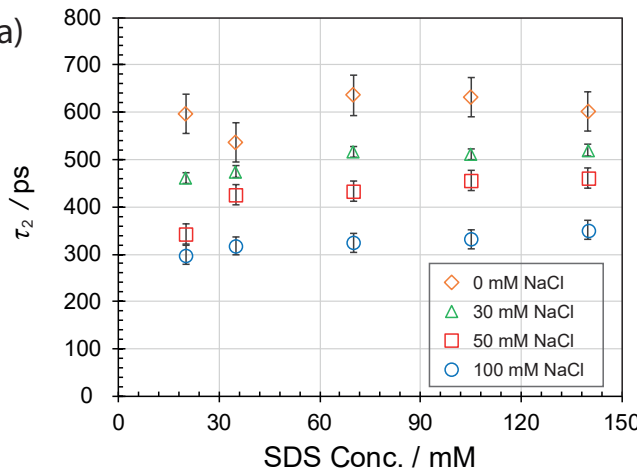

(c)

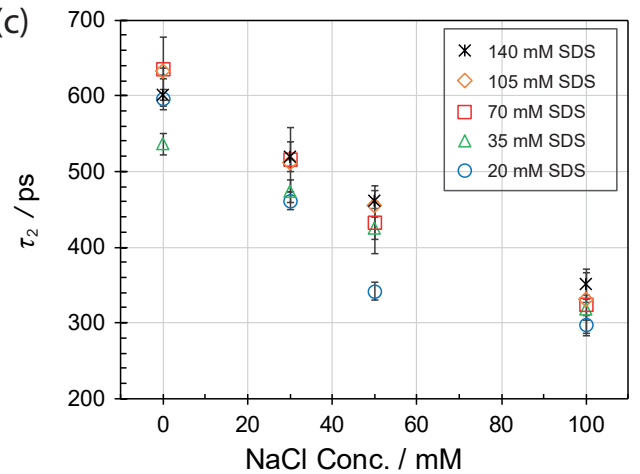

(b)

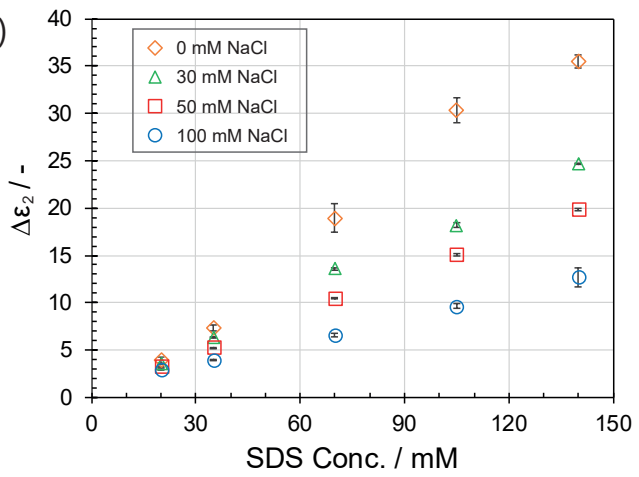

(d)

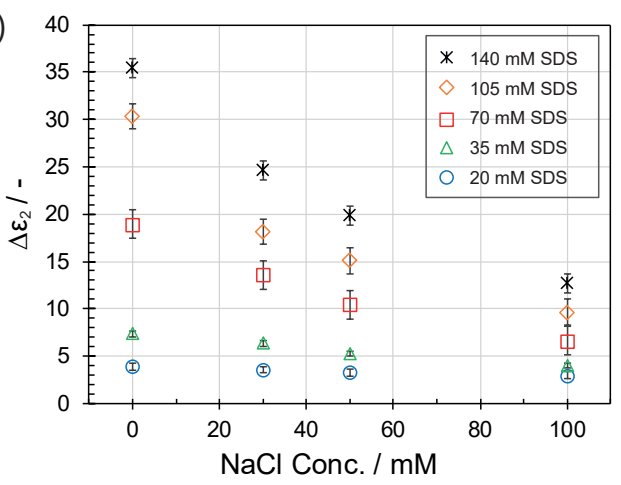

FIGURE 4: The effect of SDS and $\mathrm{NaCl}$ concentration on the second relaxation mode: (a) relaxation time $\tau_{2}$ versus SDS concentration; (b) relaxation amplitude $\Delta \varepsilon_{2}$ versus SDS concentration; (c) relaxation time $\tau_{2}$ versus $\mathrm{NaCl}$ concentration; (d) relaxation amplitude $\Delta \varepsilon_{2}$ versus $\mathrm{NaCl}$ concentration. 
is, to a good approximation, a linear increase of $\Delta \varepsilon_{2}$ with an increase in surfactant concentration. These changes in $\tau_{2}$ and $\Delta \varepsilon_{2}$ are analyzed by means of a physicochemical model in the discussion section. Figures 4(c) and 4(d) reveal the effect of the $\mathrm{NaCl}$ content, at a given SDS concentration, on $\tau_{2}$ and $\Delta \varepsilon_{2}$, respectively. Generally, we find that adding $\mathrm{NaCl}$ to the samples results in a decrease of $\tau_{2}$ and $\Delta \varepsilon_{2}$ and this decrease is more or less linear. In terms of $\tau_{2}$, the slopes are similar for all the different surfactant concentrations. In terms of $\Delta \varepsilon_{2}$, the influence is more evident at higher concentrations of SDS and there is a considerable decline when $\mathrm{NaCl}$ is added. At low surfactant concentrations, a drop in $\Delta \varepsilon_{2}$ is observed as well, but to a much lower extend. These effects are also analyzed in more detail in the next section.

\section{Discussion}

In this section, we interpret our experimental results based on a model developed for the relaxation processes around spherical colloidal particles. We expand this model and combine it with other correlations for SDS surfactant solutions.

\subsection{Micelle Relaxation Model}

Grosse and Foster developed a model to explain the permittivity change of a suspension consisting of charged colloidal particles of spherical shape in aqueous electrolytes $[20,21,36]$. This model incorporates two, a low and a high frequency relaxation process of the colloidal particles (i.e., $i=1$ and 2), and it was also successfully applied to describe the micellar relaxations in micellar surfactant solutions $[14,17]$.

For the application of the Grosse and Foster model, it is assumed that the micelles consist of a spherical hydrophobic core with radius $R_{m i c}$, static permittivity $\varepsilon_{p}$, conductivity $\sigma_{p}$, and they are dispersed in a medium of static permittivity $\varepsilon_{m}$ and conductivity $\sigma_{m}$; a schematic is shown in Figure 5. The charge of the micelle attracts and repels counter-ions and co-ions from the electrolyte, respectively; hence, an EDL is formed. The Stern and diffuse layer of the EDL are specified with dashed lines around the micelle in Figure 5. In addition, the first and second relaxation processes are indicated with radial and tangential arrows, respectively. The presence of the EDL is captured by the micelle surface conductivity $\lambda_{s}$ and related to the fact that the conductivity (ion composition) in the EDL differs to that of the bulk. Applying the Grosse and Foster model to the micellar system, the high frequency relaxation time $\tau_{2}$ can be written as

$$
\tau_{2}=\frac{\varepsilon_{0} \varepsilon_{m}\left(\frac{\varepsilon_{p}}{\varepsilon_{m}}+2\right)}{\sigma_{m}(2 D u+2)}
$$

Here, we deviate from the original approach and introduced the Dukhin number $D u=\frac{\lambda_{s}}{R_{m i c} \sigma_{m}}$ which is a 


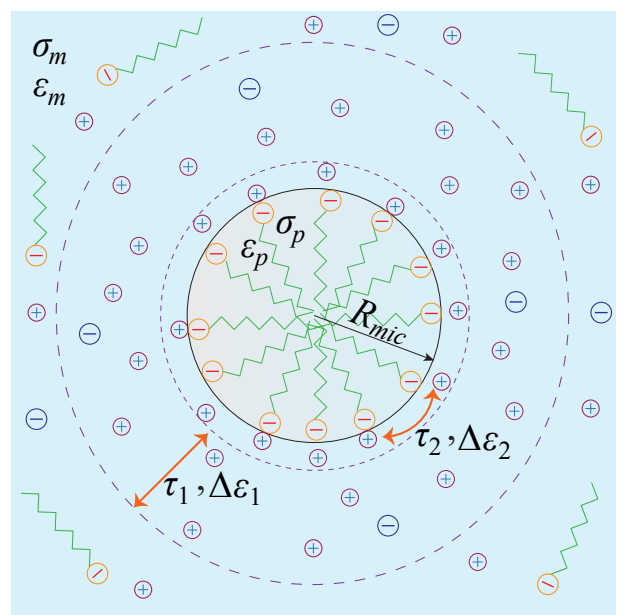

FIGURE 5: The structure of EDL around the micelle

dimensionless group that describes the relative importance of the surface conductivity to a phenomenon that affects the medium conductivity [37]. Likewise, based on the Grosse and Foster model, the corresponding permittivity amplitude of the relaxation $i=2$ can be expressed as

$$
\Delta \varepsilon_{2}=9 \frac{\nu_{m i c} \varepsilon_{m}}{\left(\frac{\varepsilon_{p}}{\varepsilon_{m}}+2\right)}\left(\frac{2 D u-\frac{\varepsilon_{p}}{\varepsilon_{m}}}{2 D u+2}\right)^{2},
$$

where $\nu_{m i c}$ is the micelle volume fraction of the suspension. For spherical micelles, it is

$$
\nu_{m i c}=\frac{4 \pi N_{A v} R_{m i c}^{3}\left(c-c_{f}\right)}{3 N_{a g}} .
$$

Here, $N_{A v}$ is the Avogadro number; $c$ is the total surfactant concentration; $c_{f}$ is the concentration of free surfactant molecules in the bulk liquid not participating in the formation of micelles; and $N_{a g}$ is the aggregation number of the micelles. The dependency of the SDS aggregation number $N_{a g}$ on the SDS and $\mathrm{NaCl}$ concentrations were investigated in several works [38-42]. We use an empirical correlation $\mathrm{N}_{a g}=K c_{N a^{+}}^{\gamma}$, that is proposed by Bales et al [38], based on the works of Huisman [43], Sasaki et al. [44] and Quina et al. [45], where $K$ and $\gamma$ are constants depending on the surfactant alkyl chain length. Bales et al. [38] obtained the constants associated with this equation and proposed the following equation to quantify the aggregation number of SDS in the presence of $\mathrm{NaCl}$ at $25^{\circ} \mathrm{C}$. It is

$$
N_{a g}=49.5\left(\frac{0.27 c+0.73 c_{f}+c_{N a C l}}{c m c_{0}}\right)^{1 / 4}
$$

where $c_{\mathrm{NaCl}}$ is the $\mathrm{NaCl}$ concentration in the suspension and $\mathrm{cmc}_{0}$ is the critical micelle concentration of SDS in the absence of $\mathrm{NaCl}$ which corresponds to $0.008 \mathrm{M}$ at this temperature. We apply this equation for a 
temperature of $22^{\circ} \mathrm{C}$ since the respective error can be considered as negligible compared to other inaccuracies. In addition, we use the implicit correlation

$$
\log \left(c_{f}\right)=-3.63-0.73 \log \left[0.27\left(c-c_{f}\right)+c_{f}+c_{N a C l}\right]
$$

that Quina et al. [45] proposed to calculate the free surfactant molecule concentrations $c_{f}$ of SDS.

In the following section, we discuss the changes in $\tau_{2}$ and $\Delta \varepsilon_{2}$ for the SDS samples with and without the addition of $\mathrm{NaCl}$. We use Equations (4)-(8) along with assuming $\varepsilon_{m}=\varepsilon_{\infty}+\Delta \varepsilon_{w}=79.5$, obtained from fitting the pure water data, $\varepsilon_{p}=2$ as well as $\sigma_{p}=0$. The reason for this procedure is that we aim to the identify the parameters that are responsible for the observed changes in relaxation time and amplitude according to the Grosse and Foster model.

\subsection{Influence of the Surfactant Concentration}

First, we would like to analyze the relaxation time. The fluctuation of $\tau_{2}$ that we observe are always less than $20 \%$ and more or less within the standard deviation of the method. We compute the $D u$ number for a certain SDS concentration from Equation (4) and use it to compute the respective ratios of $\lambda_{s} / R_{m i c}$. The values, along with $\sigma_{m}$, are plotted versus the SDS concentration in Figure 6. The $D u$ number reduces from 14.1 for a $20 \mathrm{mM}$ SDS sample to 2.2 for an $140 \mathrm{mM}$ SDS sample. The accompanying change of $\lambda_{s} / R_{\text {mic }}$ is negligible compared to the one order of magnitude increase in the medium conductivity $\sigma_{m}$. Hence, we note that the Dukhin number is mainly determined by the increase of the medium conductivity when the surfactant concentration increases. However, the change in the relaxation time $\tau_{2}$, according to Equation (4), does not depend on the single parameters but on their combination in the form of $\sigma_{m}(2 D u+2)$. Figure 6 clarifies that this combination does not significantly change and, hence, this explains why $\tau_{2}$ is hardly affected by the change in surfactant concentration.

We use the similar approach to analyze the more or less linear increase of $\Delta \varepsilon_{2}$ that is observed when the surfactant concentration increases. According to Equation (5), the amplitude is proportional to the surfactant volume fraction or the dimensionless group $A=\left(\frac{2 D u-\frac{\varepsilon_{p}}{\varepsilon_{m}}}{2 D u+2}\right)^{2}$. Figure 6 shows that the parameter $A$ only slightly decreases when the surfactant concentration increases over the investigated range; the change is within an order of magnitude. The accompanying micelle volume fraction increases by one order of magnitude. Consequently, we conclude that the increase in $\Delta \varepsilon_{2}$ is directly triggered by the increase of $\nu_{m i c}$.

From a physicochemical point of view, the added surfactant rather participates in micelle formation than in dissociation in the bulk. Based on the insights we gained above, the surface conductivity then remains almost 


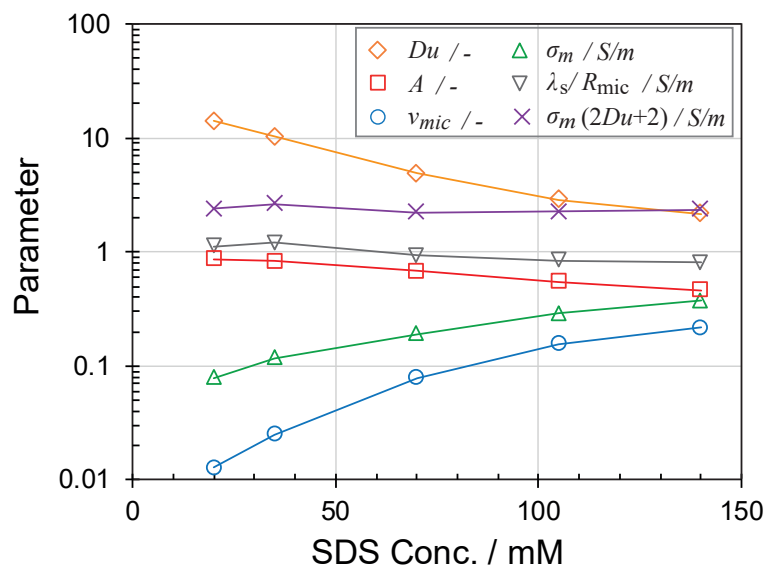

FIGURE 6: Parameters affecting $\tau_{2}$ and $\Delta \varepsilon_{2}$ versus SDS concentration. Lines are guide to the eye.

the same while the increase in medium conductivity can be attributed to the larger number of charged micelles present within the system. The relaxation time is mainly related to the properties of the EDL which, according to the Dukhin number, does not change significantly. However, the relaxation amplitude is a measure for the loss of energy in the system. With a higher population of micelles, the dissipation of energy increases as it can be seen by the increase of the relaxation amplitude.

\subsection{Influence of the Electrolyte Addition}

As mentioned before, the difference between the DRS of the samples with and without $\mathrm{NaCl}$ lies in the micellar relaxation $(i=2)$. The addition of $\mathrm{NaCl}$ decreases both $\Delta \varepsilon_{2}$ and $\tau_{2}$ and we use Equations (4)-(8) to analyze these observations. To do so, we choose an exemplary data set with an SDS content of $70 \mathrm{mM}$ and varying $\mathrm{NaCl}$ concentrations to explain the behavior.

According to Equation (4), the relaxation time is proportional to $1 / \sigma_{m}$ and $1 / D u$. Figure 7 shows the behavior of the parameters directly and indirectly related to the change in $\tau_{2}$ as a function of the electrolyte concentration. The most significant changes are found for the medium conductivity $\sigma_{m}$, which increases by one order of magnitude, and the $D u$ number, which decreases by one order of magnitude, to good approximation. Since the ratio $\lambda_{s} / R_{m i c}$ is hardly influenced by the $\mathrm{NaCl}$ content, the change in $D u$ number is solely related to the change of the medium conductivity. Consequently, the denominator $\sigma_{m}(2 D u+2)$ in Equation (4) only slightly increases over the $\mathrm{NaCl}$ concentration and we can conclude that the drop of $\tau_{2}$ can be attributed to the increase of medium conductivity when electrolyte is added.

The amplitude $\Delta \varepsilon_{2}$ decreases when the $\mathrm{NaCl}$ concentration increases. According to Equation (5), an increase of the micelle volume fraction $\nu_{m i c}$ could trigger this behavior but this parameter remains more or less constant over the $\mathrm{NaCl}$ concentration, cf. Figure 7. However, the other defining parameter $A$ decreases 


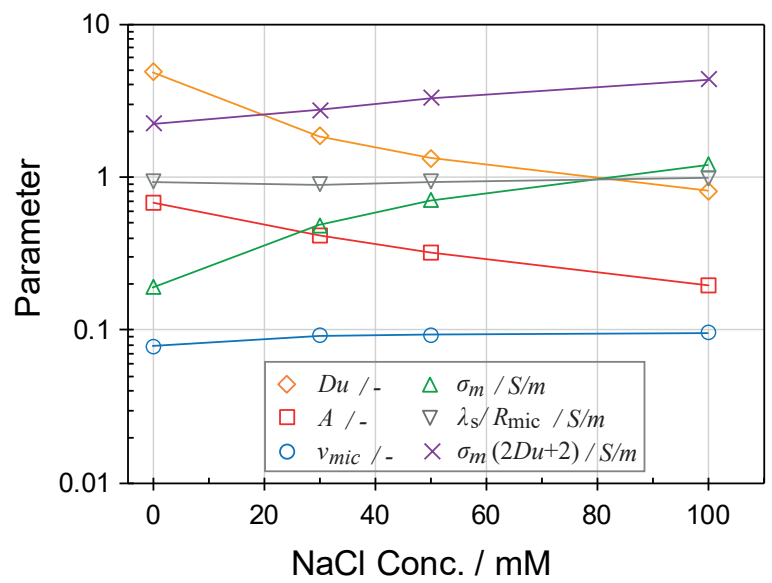

FIGURE 7: Parameters affecting $\tau_{2}$ and $\Delta \varepsilon_{2}$ versus $\mathrm{NaCl}$ concentration. Lines are guide to the eye.

due to the decrease in the $D u$ number which explains the behavior of $\Delta \varepsilon_{2}$ when $\mathrm{NaCl}$ is added.

We can conclude that the the micellar relaxation process and the electrolyte concentration are linked by the characteristics of the EDL around the micelle. The addition of $\mathrm{NaCl}$ increases both, the bulk and the surface conductivity, where the increase in medium (bulk) conductivity is more pronounced which reduces the Dukhin number. The higher bulk conductivity lowers the electric field strength in the vicinity of the micelle. Likewise, the increase in surface conductivity (ion concentration in the EDL) lowers the effective dipole moment of the micelle by shielding. Both phenomena result in a reduced relaxation amplitude.

In addition, the decrease in the relaxation time by adding more electrolyte can be physically attributed to the stagnant layer of the EDL. Increase in surface conductivity by adding $\mathrm{NaCl}$ is related to the higher concentration of counter-ions in this region. This denser region of counter-ions can keep up faster with the change in the AC the electrical field [46]. Therefore, the relaxation process happens at higher frequency and lower relaxation times, respectively.

\subsection{Aggregation Number and Dukhin Number}

In the previous section, we clarify that the Dukhin number of the micelle has a major influence on the second relaxation process but it is usually not readily available. Hence, we infer a general correlation of the Dukhin number for an aqueous suspension of SDS and $\mathrm{NaCl}$ in this section.

The concentrations of surfactant and electrolyte determine the bulk conductivity as well as the micelle size and the charge in the EDL; all these parameter are included in the $D u$ number. We propose an expression similar to the aggregation number, and assume that the $D u$ number can be solely expressed with the concentration of counter-ions (sodium ions) according to $D u=K^{\prime} c_{\mathrm{Na}^{+}}^{\gamma^{\prime}}$, where $K^{\prime}$ and $\gamma^{\prime}$ are con- 


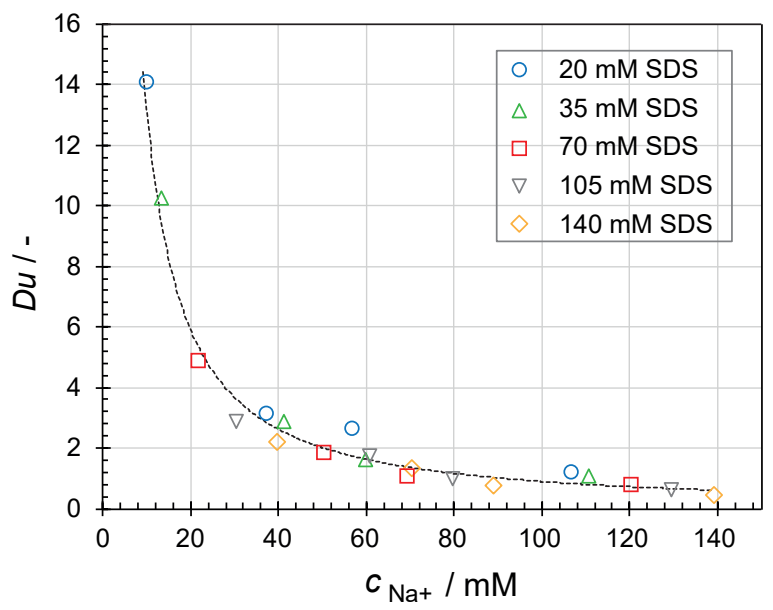

FIGURE 8: Dukhin Number versus sodium ion concentration. Symbols are data extracted from DRS measurements while dashed line is the respective regression.

stants specific to the electrolyte and surfactant combination. We test our hypothesis by plotting the $D u$ numbers that we extracted from the DRS data versus the concentration of the sodium ions in the solutions. The sodium ion concentration has contributions from the added electrolyte and the dissociated surfactant heads; it is $c_{N a^{+}}=0.27 c+0.73 c_{f}+c_{N a C l}$ which is also utilized in Equation (7). The value 0.27 is in fact, the apparent degree of counter-ion dissociation [38].

Figure 8 shows the extracted $D u$ numbers for the so-computed sodium ion concentration of the suspensions. Note again that the data includes a wide range of SDS and $\mathrm{NaCl}$ concentrations. We strikingly observe that, despite the large range of different surfactant concentrations, all $D u$ numbers collapse on a single curve when we express them in terms of a sodium ion concentration. The lowest sodium ion concentration arises from the pure surfactant solution with $20 \mathrm{mM}$ SDS and gives $D u$ number of around 14. Any addition of sodium ions, either in form of SDS or $\mathrm{NaCl}$, considerably lowers the $D u$ number. A respective regression results in

$$
D u=0.0574 c_{N a^{+}}{ }^{-1.186}
$$

with a very high coefficient of determination of $R^{2}=0.95$.

\subsection{Zeta Potential of SDS Micelles}

In this section, we use the micelle surface conductivities ( $D u$ numbers) that are extracted from the DRS measurements to infer the zeta potential of the SDS micelles for different surfactant and electrolyte concentrations. In detail, Bikerman in 1933 (as cited in Ref. [47]) derived the surface conductivity from a diffuse double layer from the analytical solution of the charge distribution at an infinite plate. This expression includes the con- 
tribution of migration and that of electroosmosis, which results in an additional mobility of the charges. It is

$$
\lambda_{s}=\sqrt{8 N_{A v} \varepsilon k_{B} T I}\left(\frac{\nu_{+}}{\operatorname{coth}\left(\frac{z e \zeta}{4 k_{B} T}\right)-1}-\frac{\nu_{-}}{\operatorname{coth}\left(\frac{z e \zeta}{4 k_{B} T}\right)+1}+\frac{4 \varepsilon k_{B} T}{z e \mu} \frac{1}{\operatorname{coth}^{2}\left(\frac{z e \zeta}{4 k_{B} T}\right)-1}\right)
$$

where $k_{B}$ is the Boltzmann constant; $T$ is the absolute temperature; $I=1 / 2 \sum z_{i}^{2} c_{i}$ is the ionic strength of the solution that can be calculated in terms of the valency $z_{i}$ and the concentration $c_{i}$ of the ionic species $i$; $\nu_{+}$and $\nu_{-}$are ion mobilities of positive and negative ion species, respectively; $e$ is the elementary charge; $\zeta$ is the zeta potential; and $\mu$ is the dynamic viscosity of the medium. In this study, we use the concentration of free sodium and dodecyl ions as well as that of the chloride to calculate the ionic strength of the medium.

There is a discrepancy in using Equation (10) since it is derived for planar surfaces. An analytical solution for the EDL around a cylindrical or spherical surface is to our knowledge not yet found, not even for the case of the Debye-Hückel linearization. Nevertheless, when the frequency of the electric field is high, the electroosmotic motion in the EDL around a sphere can be considered as a local process with a very short range [48]. The surface conductivities derived in this work are measured at very high frequencies and we consequently assume that there is no influence of the (spherical) geometry of the EDL. Using Equation (10) also requires the values of the ion mobilities $\nu_{i}$. Here, we engage the Nernst-Einstein correlation $D_{i}=$ $\nu_{i} k_{B} T /\left(z_{i} e\right)$ along with the diffusivity $D_{i}$ data of $1.334 \times 10^{-9}, 2.032 \times 10^{-9}$, and $0.639 \times 10^{-9} \mathrm{~m}^{2} / \mathrm{s}$ for sodium, chloride and dodecyl sulfate ions, respectively [49]. For the calculation of the zeta potential in the absence of $\mathrm{NaCl}$, the mobilities of sodium and dodecyl sulfate ions are used. However, for samples containing $\mathrm{NaCl}$ we use only the sodium ion and chloride mobilities since they are 3 to 4 times higher compared to the dodecyl sulfate. Additionally, the majority of the dodecyl sulfate ions aggregates to form the micelle and, thus, does hardly contribute to the diffuse double layer.

The computed zeta potentials of the SDS micelles as a function of the surfactant content are plotted in Figure 9 for various concentrations of $\mathrm{NaCl}$. As can be seen in this figure, all zeta potentials are negative. For a given $\mathrm{NaCl}$ concentration, the relationship between zeta potential and surfactant concentration is linear to a good approximation. Any addition of surfactant decreases the magnitude of the zeta potential. This is mainly related to the increase in free sodium ion concentration which accordingly increases the ionic strength of the medium while the surface conductivity remains more or less constant. For example, the zeta potential for samples without $\mathrm{NaCl}$ changes from $-120 \mathrm{mV}$ for $20 \mathrm{mM}$ SDS to $-95 \mathrm{mV}$ for $140 \mathrm{mM}$ SDS. The addition of $\mathrm{NaCl}$ to the samples also decreases the absolute values of the micelle's zeta potential. This decrease is mainly related to shielding of the surface charge by the electrolyte counter-ion $[50,51]$. 


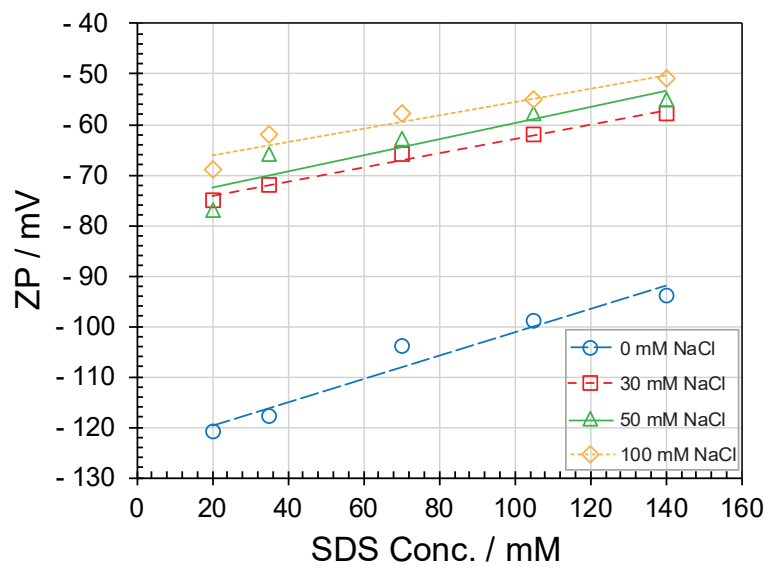

FIGURE 9: Zeta potential of the SDS micelles versus the SDS concentration for different concentrations of $\mathrm{NaCl}$. Lines are linear regressions.

The comparison of our results with literature data shows very good agreement. Stigter and Mysels [52] measured the electrophoretic mobility of SDS solutions using a tracer for different concentrations of $\mathrm{NaCl}$. They reported zeta potential values of about -120 to $-70 \mathrm{mV}$ for 0 and $100 \mathrm{mM}$ of $\mathrm{NaCl}$ at $\mathrm{CMC}$. In addition, Dunstan and White [53] developed a theoretical approach to infer the electrophoretic mobility of a micellar SDS suspension based on conductivity measurements. They reported $-143 \mathrm{mV}$ and $-72 \mathrm{mV}$ at $\mathrm{CMC}$ and $\mathrm{NaCl}$ concentrations of 0 and $100 \mathrm{mM}$, respectively. The agreement of these values and our data proves that DRS of a micellar system, along with the implemented model, is a reliable and accurate method to infer the zeta potential of a micelle.

\section{Summary}

In this work, we use dielectric relaxation spectroscopy (DRS) to study micellar solutions of sodium dodecyl sulfate with and without addition of sodium chloride. Although no attempt was found in literature, studying the effect of electrolyte addition is important since it is usually the case for surfactant solutions. In addition, we employ DRS measurements along with models to infer the zeta potential of micelles; the main parameter of all electrokinetic phenomena. In this regard, DRS is an extremely fast and reliable method that is applicable to any sample regardless of its polarity, viscosity, concentration, and turbidity. Compared to Laser Doppler Electrophoresis, another standard method for electrokinetic measurements, it is therefore far more robust and flexible in terms of samples that can be processed.

We examine the data in a frequency range of $200 \mathrm{MHz}$ to $14 \mathrm{GHz}$ using the Cole-Cole model. Two relaxation processes are identified. The first relaxation process is related to the tangential motion of ions that 
are located in the electrical double layer around the micelles. The relaxation time of this dispersion step is hardly influenced by a change in surfactant concentration. However, we observe that the addition of $\mathrm{NaCl}$ results in a decrease of the relaxation time. The relaxation amplitude increases by adding more surfactant but decreases by adding $\mathrm{NaCl}$. The second relaxation process is related to the hydrogen bond of the water molecules which is hardly affected in the surfactant and $\mathrm{NaCl}$ concentration ranges studied in this work.

We explain the change in relaxation time and amplitude of the tangential micellar relaxation process using the Grosse and Foster model along with other correlations. Accordingly, the addition of surfactant increases the volume fraction of the micelles which results in an energy loss expressed by a increase of the relaxation amplitude. The decrease in relaxation time with the addition of $\mathrm{NaCl}$ is related to the increase in medium conductivity which lowers the electric field strengths in the vicinity as well as the effective dipole moment of the micelle. The accompanying decrease in relaxation amplitude is related to the increase in surface conductivity of the micelles.

Based on the DRS results, we infer the Dukhin number of the micellar suspension without the need of knowing its single parameters; i.e., the surface conductivity, medium conductivity, and the micelle radius. We observe that the Dukhin numbers, extracted from all different suspensions, collapse on a single curve when plotted versus the sodium ion concentration and a respective power law relationship is proposed.

Finally, we use the Bikerman equation for the surface conductivity of an EDL. Based on the Dukhin number that we extracted from the DRS data, and along with other material parameter, we calculate the zeta potential of the micelles for various surfactant and $\mathrm{NaCl}$ concentrations. The obtained zeta potential values are in very good agreement with values that are reported in literature which proves the validity of our approach.

\section{Acknowledgements}

The authors thank E.I. DuPont Canada Co. and NSERC for financial support.

\section{Conflict of Interest}

The authors declare no conflict of interest.

\section{References}

[1] Grosse, C. and Delgado, A.V., Curr. Opin. Colloid Interface Sci. 2010, 15, 145-159.

[2] Buchner, R., Pure Appl. Chem. 2008, 80, 1239-1252. 
[3] Buchner, R. and Hefter, G., Phys. Chem. Chem. Phys. 2009, 11, 8984.

[4] Shikata, T. and Imai, S.-I., Langmuir 1998, 14, 6804-6810.

[5] Rosen, L.A. and Saville, D.A., Langmuir 1991, 7, 36-42.

[6] Feldman, Y., Ram, G., and Ram, G., IEEE Trans. Dielectr. Electr. Insul. 2003, 10, 728-753.

[7] Goodwin, J.W., Colloids and Interfaces with Surfactants and Polymers - An Introduction, John Wiley \& Sons Ltd, 2004.

[8] Vaisman, L., Wagner, H.D., and Marom, G., Adv. Colloid Interface Sci. 2006, 128-130, 37-46.

[9] Cao, J., Dun, W., and Qu, H., Electrophoresis 2011, 32, 408-413.

[10] Kim, J.W., Lee, D., Shum, H.C., and Weitz, D.A., Adv. Mater. 2008, 20, 3239-3243.

[11] Wuzhang, J., Song, Y., Sun, R., Pan, X., and Li, D., Electrophoresis 2015, 36, 2489-2497.

[12] Righetti, P.G., Gelfi, C., Verzola, B., and Castelletti, L., Electrophoresis 2001, 22, 603-611.

[13] Lanzi, L., Carlà, M., Lanzi, L., and Gambi, C.M.C., J. Colloid Interface Sci. 2009, 330, $156-162$.

[14] Fernandez, P., Schrödle, S., Buchner, R., and Kunz, W., ChemPhysChem 2003, 4, 1065-1072.

[15] Barchini, R. and Pottel, R., J. Phys. Chem. 1994, 98, 7899-7905.

[16] Buchner, R., Baar, C., Fernandez, P., Schrödle, S., and Kunz, W., J. Mol. Liq. 2005, 118, $179-187$.

[17] Baar, C., Buchner, R., and Kunz, W., J.Phys.Chem.B 2001, 105, 2906-2913.

[18] Baar, C., Buchner, R., and Kunz, W., J.Phys.Chem.B 2001, 105, 2914-2922.

[19] Itatani, S. and Shikata, T., Langmuir 1997, 17, 6841-6850.

[20] Grosse, C. and Foster, K.R., J. Phys. Chem. 1987, 91, 3073-3076.

[21] Grosse, C., J. Phys. Chem. 1988, 92, 3905-3910.

[22] Buchner, R., Hölzl, C., Stauber, J., and Barthel, J., Phys. Chem. Chem. Phys. 2002, 4, 2169-2179.

[23] Ro, C. and Mikkelsen, K.V., J. Chem. Phys 1997, 107, 5319-5331.

[24] Koeberg, M., Wu, C.C., Kim, D., and Bonn, M., Chem. Phys. Lett. 2007, 439, 60-64.

[25] Buchner, R., Hefter, G.T., and May, P.M., J. Phys. Chem. A 1999, 103, 1-9. 
[26] Aleiner, G.S. and Us'yarov, O.G., Colloid J. 2010, 72, 588-594.

[27] Khademi, M., Wang, W., Reitinger, W., and Barz, D.P., Langmuir 2017, 33, 10473-10482.

[28] George, D.K., Charkhesht, A., Hull, O.A., Mishra, A., Capelluto, D.G., Mitchell-Koch, K.R., and Vinh, N.Q., J. Phys. Chem. B 2016, 120, 10757-10767.

[29] Imai, S., Shiokawa, M., and Shikata, T., J.Phys.Chem.B 2001, 105, 4495-4502.

[30] Barthel, J.M.G. and Buchner, R., Pure Appl. Chem. 1991, 63, 1473-1482.

[31] Buchner, R., Barthel, J., and Stauber, J., Chem. Phys. Lett. 1999, 306, 57-63.

[32] Nörtemann, K., Hilland, J., and Kaatze, U., J. Phys. Chem. A 1997, 101, 6864-6869.

[33] Lyashchenko, A.K. and Zasetsky, A.Y., J. Mol. Liq. 1998, 77, 61-75.

[34] Peyman, A. and Gabriel, C., Bioelectromagnetics 2007, 28, 264-274.

[35] Ono, Y. and Shikata, T., J. Phys. Chem. B 2005, 109, 7412-7419.

[36] Grosse, C. and Foster, K.R., J. Phys. Chem. 1987, 91, 6415-6417.

[37] Delgado, A.V., González-Caballero, F., Hunter, R.J., Koopal, L.K., and Lyklema, J., J. Colloid Interface Sci. 2007, 309, 194-224.

[38] Bales, B.L., Messina, L., Vidal, A., Peric, M., and Nascimento, O.R., J.Phys.Chem.B 1998, 102, 1034710358.

[39] Bales, B. and Almgren, M., J. Phys. Chem. 1995, 99, 15153-15162.

[40] Bezzobotnov, V.Y., Borbely, S., Cser, L., Farago, B., Gladkih, I.A., Ostanevich, Y.M., and Vass, S., J. Phys. Chem. 1988, 92, 5738-5743.

[41] Gehlen, M.H. and Schryver, F.C.D., J. Phys. Chem. 1993, 97, 11242-11248.

[42] Croonen, Y., Gelade, E., Zegel, M.V.D., M. Van der Auweraer, Vandendriessche, H., Schryver, F.C.D., and Almgren, M., J. Phys. Chem. 1983, 87, 1426-1431.

[43] Huisman, H.F., Light scattering of solutions of ionic detergents, Noord-Holl. Uitg. Maatsch. 1964.

[44] Sasaki, T., Hattori, M., Sasaki, J., and Nukina, K., Bull. Chem. Soc. Jpn. 1975, 48, 1397-1403.

[45] Quina, F.H., Nassar, P.M., Bonilha, J.B., and Bales, B.L., J. Phys. Chem. 1995, 99, 17028-17031. 
[46] Carrique, F., Zurita, L., and Delgado, A., Colloids Surf., A 1994, 92, 9-21.

[47] Bikerman, J.J., Kolloid-Zeitschrift 1935, 72, 100-108.

[48] Bellini, T. and Mantegazza, F., Delgado AV, editor. Interfacial Electrokinetics and Electrophoresis 2002, $401-441$.

[49] Haynes, W., Lide, D., and Bruno, T., CRC Handbook of Chemistry and Physics, 94th Edition, CRC Press, 94th edition 2016.

[50] Falahati, H., Wong, L., Davarpanah, L., Garg, A., Schmitz, P., and Barz, D.P., Electrophoresis 2014, 35, 870-882.

[51] Barz, D.P.J., Vogel, M.J., and Steen, P.H., Langmuir 2009, 25, 1842-1850.

[52] Stigter, D. and Mysels, K.J., J. Phys. Chem. 1955, 59, 45-51.

[53] Dunstan, D.E. and White, L.R., J. Colloid Interface Sci. 1990, 134, 147-151. 